\title{
Successful Strategy for High Degree of Freedom Crystal Structure Determination from Powder X-Ray Diffraction Data: A Case Study for Selexipag Form I with 38 DOF
}

\author{
Michal Hušák, ${ }^{1 *}$ Alexandr Jegorov, ${ }^{2}$ Jiri Czernek, ${ }^{3}$ Jan Rohlíček, ${ }^{1}$ Simona Žižková, ${ }^{1}$ Pavel Vraspír, ${ }^{4}$ Pavel \\ Kolesa, ${ }^{4}$ Andrew Fitch, ${ }^{5}$ and Jiri Brus ${ }^{3 *}$ \\ ${ }^{1}$ Department of Solid State Chemistry, University of Chemical Technology Prague, 16628 Prague 6, Czech \\ Republic \\ ${ }^{2}$ Teva Pharmaceuticals CR, s.r.o, Branišovská 31,370 05 České Budějovice, Czech Republic \\ ${ }^{3}$ Institute of Macromolecular Chemistry, Czech Academy of Sciences, Heyrovský sq. 2, 16206 Prague 6, Czech \\ Republic \\ ${ }^{4}$ Teva Pharmaceuticals CR, s.r.o, Ostravská 29, 74770 Opava-Komárov, Czech Republic \\ ${ }^{5}$ ID22, Structure of Materials Group, ESRF- The European Synchrotron, CS40220, F-38043 Grenoble Cedex 9 , \\ France
}

AUTHOR EMAIL ADDRESSES: Michal.Husak@vscht.cz, Alexandr.Jegorov@tevapharm.cz, czernek@imc.cas.cz, Jan.Rohlicek@vscht.cz, zizkovas@vscht.cz,Pavel.Vraspir@tevapharm.cz, pavel.kolesa@tevapharm.cz, fitch@esrf.fr, brus@imc.cas.cz

\section{Table of Content}

Supporting Information S1 - Solid-State NMR Spectroscopy

Supporting Information S2 - Periodic Computational Methods used for Direct Structure Verification

Supporting Information S3 - Details of the crystal structure of Selexipag Form I

Supporting Information S4 - Linear regression models of the experimentally determined isotropic chemical shifts and DFT-calculated shielding data

Supporting Information S5 - Results of the analysis of the ${ }^{1} \mathrm{H}-{ }^{13} \mathrm{C}$ HETCOR spectrum of selexipag 
1D MAS NMR experiments: All solid-state NMR spectra were measured at $11.7 \mathrm{~T}$ using a Bruker Avance 500 WB/US NMR spectrometer (2013) in a double-resonance 4-mm probe-head at spinning frequencies 10-12 kHz.

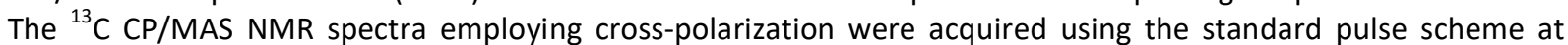
spinning frequency of $11 \mathrm{kHz}$. The recycle delay was $4 \mathrm{~s}$ and the cross-polarization contact time was 2 ms. The strength of spin-locking fields $B_{1}\left({ }^{13} \mathrm{C}\right)$ expressed in frequency units $\omega_{1} / 2 \pi=\gamma B_{1}$ was $64 \mathrm{kHz}$. The spectra were referenced to $\alpha$-glycine (176.03 ppm). The ${ }^{1} \mathrm{H}$ CRAMPS NMR spectra with DUMBO homodecoupling were measured at $10 \mathrm{kHz}$ (MAS frequency) and number of scans 16-64. The $90^{\circ}\left({ }^{1} \mathrm{H}\right)$ pulse-length was $2.2 \mu \mathrm{s}$, power level for DUMBO shape pulse was $71 \mathrm{~W}$, DUMBO pulse length $32 \mu$ s and the number of loops for digital averaging was 4-8. All parameters were optimized on glycine to reach maximum spectral resolution $\left(\Delta v\left(\mathrm{NH}_{3}{ }^{+}\right)=250 \mathrm{~Hz}\right.$ and $\Delta v\left(\mathrm{CH}_{2}\right)=230 \mathrm{~Hz}$ ). The ${ }^{1} \mathrm{H}$ scale was calibrated with external standard - alanin (low-field $\mathrm{NH}_{3}$ signal at $8.5 \mathrm{ppm}$ and the high field $\mathrm{CH}_{3}$ signal at $1.2 \mathrm{ppm}$.

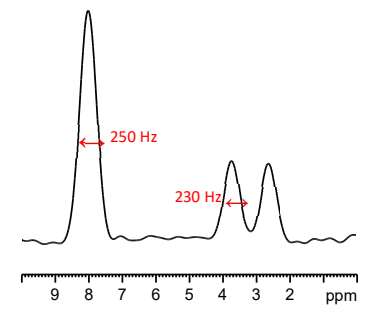

Figure $\mathrm{S} 1 .{ }^{1} \mathrm{H}$ CRAMPS NMR spectrum $(10 \mathrm{kHz})$ of glycine.

2D ${ }^{1} \mathrm{H}-^{13} \mathrm{C}$ FSLG HETCOR MAS NMR experiments: Two-dimensional (2D) ${ }^{1} \mathrm{H}-^{13} \mathrm{C}$ HETCOR experiments were performed using the FSLG (Frequency Switched Lee-Goldburg) decoupling during the $t_{1}$ evolution period consisting of 64-128 increments each made of 128-440 scans with a dwell time of $42.6 \mu$ s (Figure S2). Rotation frequency was $\omega_{\mathrm{r}} / 2 \pi=12 \mathrm{kHz}$. The $B_{1}\left({ }^{1} \mathrm{H}\right)$ field strength of FSLG and SPINAL-64 decoupling expressed in frequency units $\omega_{1} / 2 \pi=\gamma B_{1}$ was $89.3 \mathrm{kHz}$.

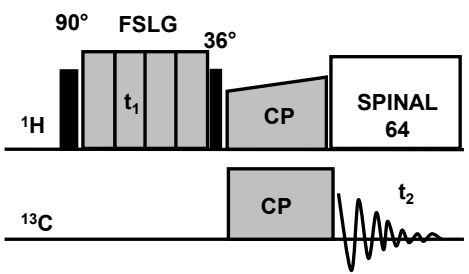

Figure S2. Schematic representation of $2 \mathrm{D}^{1} \mathrm{H}-{ }^{13} \mathrm{C}$ FSLG HETCOR MAS NMR experiment

${ }^{1} \mathrm{H}-{ }^{1} \mathrm{H}$ DQ/SQ DUMBO NMR correlation experiments: The $2 \mathrm{D}{ }^{1} \mathrm{H}-{ }^{1} \mathrm{H}$ DQ/SQ DUMBO NMR correlation spectra were measured using the ${ }^{1} \mathrm{H}-{ }^{1} \mathrm{H}$ double-quantum (DQ) experiment employing the SPC5 recoupling sequence at spinning frequency $\omega_{\mathrm{r}} / 2 \pi=10 \mathrm{kHz}$ (Figure S3). The recycle delay was $4 \mathrm{~s}, t_{1}$ evolution period consisted of 128 increments each made of 64 scans. The DQ coherence excitation and reconversion consisted of 1-4 loops (duration of one loop was $40 \mu \mathrm{s})$. The DUMBO decoupling was applied during both detection periods.

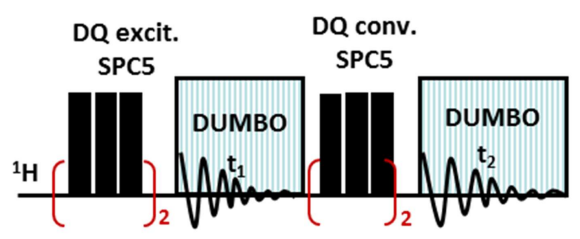

Figure S3. Schematic representation of $2 \mathrm{D}^{1} \mathrm{H}-{ }^{1} \mathrm{H} D Q / S Q$ DUMBO NMR experiment with SPC5recoupling sequence. 
Supporting Information S2 - Periodic Computational Methods used for Direct Structure Verification (convergence criteria and final values)

Table S1. DFT-D Convergence Criteria and the Obtained Results

\begin{tabular}{ccccc}
\hline & $\begin{array}{c}\text { Energy change } \\
(\mathrm{eV} / \text { atom })\end{array}$ & $\begin{array}{c}\text { Maximal force }(\mathrm{eV} / \\
\AA\end{array}$ & $\begin{array}{c}\text { Maximal } \\
\text { displacement }(\AA)\end{array}$ & $\begin{array}{c}\text { Maximal stress } \\
(\mathrm{GPa})\end{array}$ \\
\hline $\begin{array}{c}\text { Fine setting } \\
\text { convergence }\end{array}$ & $1.0 \times 10^{-5}$ & $3.0 \times 10^{-2}$ & $1.0 \times 10^{-3}$ & $5.0 \times 10^{-2}$ \\
limit & & & & \\
Fixed cell run & $9.09 \times 10^{-8}$ & $2.18 \times 10^{-2}$ & $2.14 \times 10^{-5}$ & $\mathrm{NA}$ \\
Free cell run & $1.28 \times 10^{-6}$ & $2.36 \times 10^{-2}$ & $4.19 \times 10^{-4}$ & $1.16 \times 10^{-2}$ \\
\hline
\end{tabular}




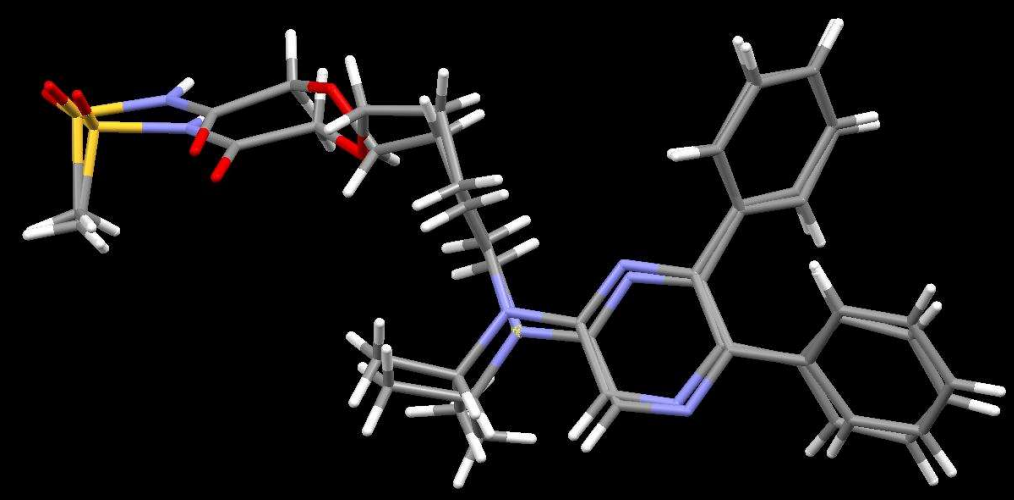

Figure S4. RMS overaly of the two molecules in the asymmetric units of Selexipag Form I.

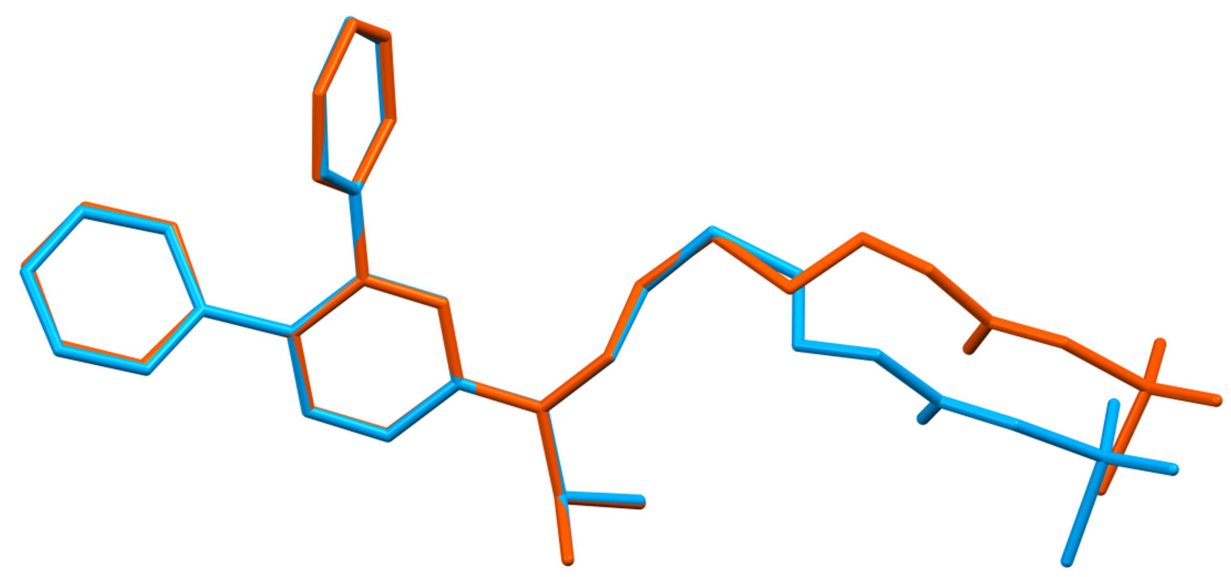

Figure S5. Overlay of both molecules in the asymmetric part of the unit cell. Orange: molecule with atomic labels C1 - O35, light blue: molecule with atomic labels $\mathrm{C} 1 \mathrm{a}$ - 035a. The flexible side-chain atoms were excluded from RMS to highlight the rigid part overlay correspondence. 
Table S2. Mogul-detected unusual torsion angles in the symmetry independent molecules of Selexipag Form I.

\begin{tabular}{llllll}
\hline Type Molecule & \multicolumn{1}{c}{ Fragment } & \multicolumn{1}{c}{ Classification } & No. of hits Query value | d(min) | Local density \\
\hline torsion I_1 & C10 C11 C8 C7 & Unusual (enough hits) 579 & 68.034 & 1.022 & 0.043 \\
torsion I_1 & O27 C26 C25 C24 & Unusual (enough hits) 1720 & 149.114 & 0.015 & 0.003 \\
torsion I_1 & O32 C29 C28 O27 & Unusual (enough hits) 328 & -17.661 & 0.006 & 0.037 \\
torsion I_2 & C12a C11a C8a C7a & Unusual (enough hits) 579 & 69.170 & 0.036 & 0.029 \\
torsion I_2 & C28a O27a C26a C25a Unusual (enough hits) 247 & -143.413 & 4.738 & 0.045 \\
torsion I_2 & O32a C29a C28a O27a Unusual (enough hits) 328 & 5.599 & 0.065 & 0.043 \\
\hline
\end{tabular}

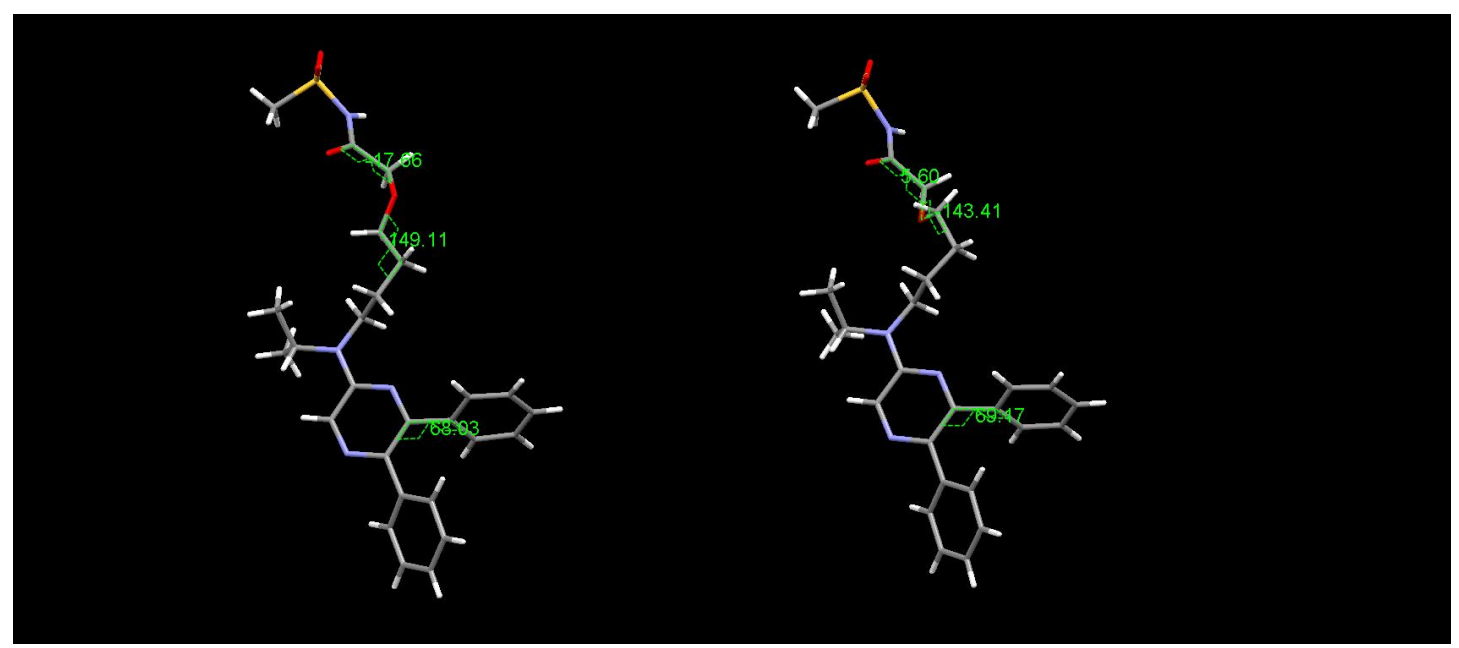

Figure S6. Vizualization of the Mogul-detected unusual torsion angles in the molecules of Selexipag Form I. 
Supporting Information S4 - Linear regression models of the experimentally determined isotropic chemical shifts and DFT-calculated shielding data

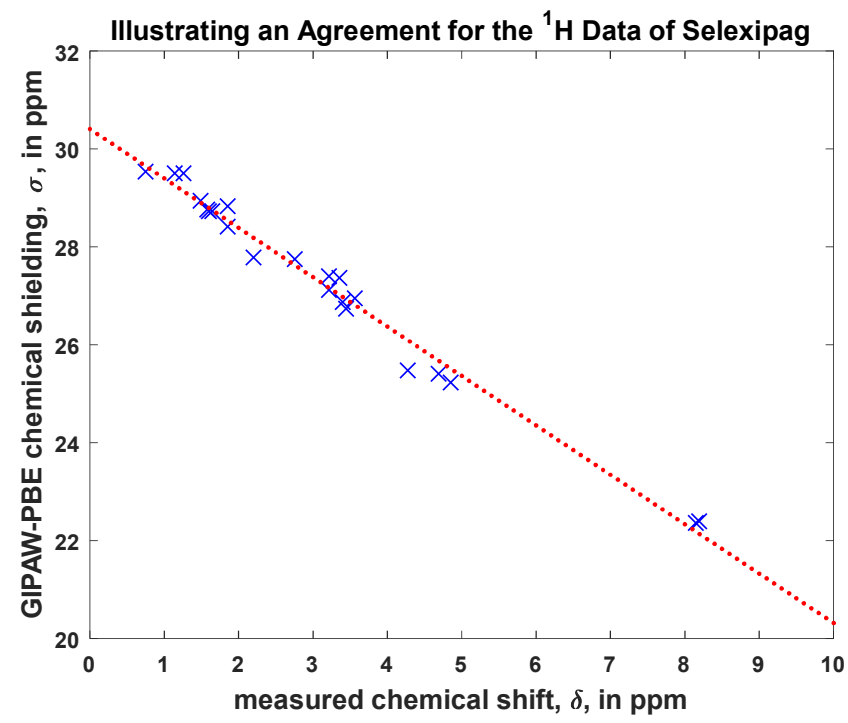

Figure S7. Linear regression plot of the experimentally determined ${ }^{1} \mathrm{H}$ NMR isotropic chemical shifts $(\delta$, ppm) and DFT-calculated ${ }^{1} \mathrm{H}$ NMR shielding data $(\sigma, \mathrm{ppm})$ obtained for Selexipag Form I. The best fit line is shown in red $\left(\sigma=-1.00881^{*} \delta+30.4058 \mathrm{ppm}\right)$.

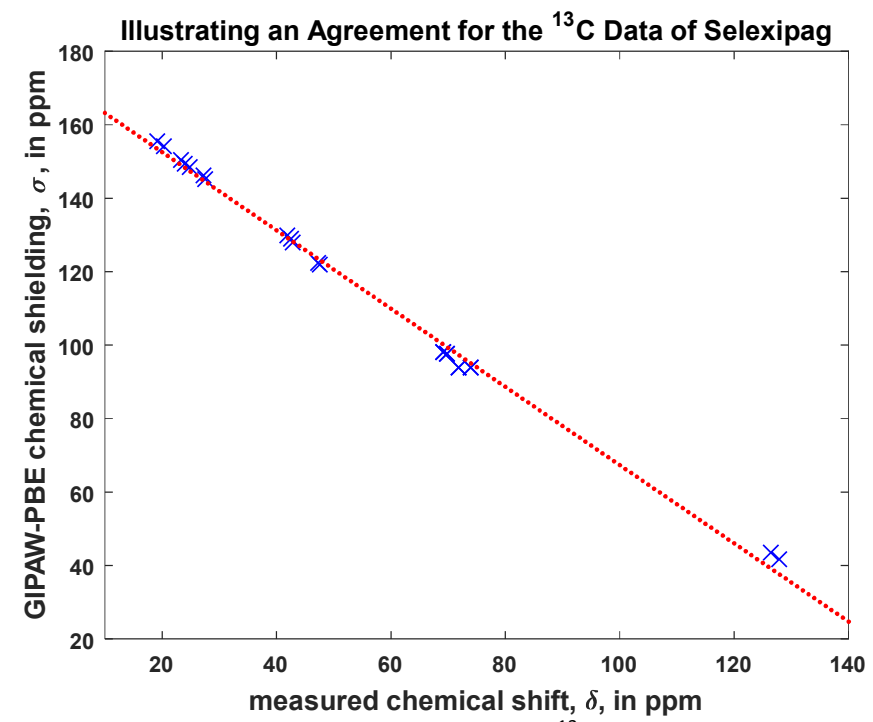

Figure S8. Linear regression plot of the experimentally determined ${ }^{13} \mathrm{C}$ NMR isotropic chemical shifts $(\delta$, ppm) and DFTcalculated ${ }^{13} \mathrm{C}$ NMR shielding data $(\sigma, \mathrm{ppm})$ obtained for Selexipag Form I. The best fit line is shown in red $\left(\sigma=-1.06546^{*} \delta+\right.$ $173.8744 \mathrm{ppm})$. 
Supporting Information S5 - Results of the analysis of the ${ }^{1} \mathrm{H}-{ }^{13} \mathrm{C}$ HETCOR spectrum of Selexipag I

Table S3. Experimentally determined isotropic ${ }^{1} \mathrm{H}$ and ${ }^{13} \mathrm{C}$ NMR chemical shifts $\delta\left({ }^{1} \mathrm{H}\right)$ and $\delta\left({ }^{13} \mathrm{C}\right)$; GIPAW-PBEcalculated chemical shielding as parsed from the CASTEP output $\sigma\left({ }^{1} \mathrm{H}\right)$ and $\sigma\left({ }^{13} \mathrm{C}\right)$; and theoretical chemical shifts $\varepsilon\left({ }^{1} \mathrm{H}\right)$ and $\varepsilon\left({ }^{13} \mathrm{C}\right)$ approximated by entering a $\sigma$ value into the corresponding models:

$\varepsilon(\mathrm{C})=-0.9348072 * \sigma(\mathrm{C})+162.7592 \mathrm{ppm} ; \varepsilon(\mathrm{H})=-0.976247 * \sigma(\mathrm{H})+29.7308 \mathrm{ppm}$ In an ideal case, $\varepsilon$ and $\delta$ would be the same for a given nucleus. ${ }^{1,2}$

\begin{tabular}{|c|c|c|c|c|c|c|c|c|}
\hline $\begin{array}{c}\text { resolved } \\
{ }^{13} \mathrm{C}-{ }^{1} \mathrm{H} \\
\text { pair \# }\end{array}$ & $\begin{array}{c}\text { carbon(s) } \\
\text { site }(s)\end{array}$ & $\begin{array}{l}\delta^{13} \mathrm{C} \\
(\mathrm{ppm})\end{array}$ & $\begin{array}{l}\sigma^{13} \mathrm{C} \\
(\mathrm{ppm})\end{array}$ & $\begin{array}{l}\varepsilon^{13} \mathrm{C} \\
(\mathrm{ppm})\end{array}$ & $\begin{array}{c}\text { proton(s) } \\
\text { site }(\mathrm{s})\end{array}$ & $\begin{array}{c}\delta^{1} \mathrm{H} \\
(\mathrm{ppm})\end{array}$ & $\begin{array}{c}\sigma^{1} \mathrm{H} \\
(\mathrm{ppm})\end{array}$ & $\begin{array}{c}\varepsilon^{1} \mathrm{H} \\
(\mathrm{ppm})\end{array}$ \\
\hline 1 & C15 "A" & 126.3414 & 43.5550 & 122.0437 & @C15 "A" & 8.20 & 22.3870 & 7.88 \\
\hline 2 & C15 "B" & 127.8400 & 41.5489 & 123.9190 & @C15 "B" & 8.15 & 22.3750 & 7.89 \\
\hline 3 & C17 "A" & 47.2834 & 122.3692 & 48.3676 & @C17 "A" & 4.68 & 25.4019 & 4.93 \\
\hline 4 & C18 "A" & 24.8684 & 148.5970 & 23.8497 & @C18 "A" & 1.57 & 28.7787 & 1.64 \\
\hline 5 & C19 "A" & 20.2940 & 154.2662 & 18.5501 & @C19 "A" & 1.61 & 28.7237 & 1.69 \\
\hline 6 & C20 "A" & 41.7864 & 129.7139 & 41.5017 & @C20 "A" & 3.32 & 27.4036 & 2.98 \\
\hline 7 & C21 "A" & 23.4623 & 150.3384 & 22.2218 & @C21 "A" & 0.51 & 29.5419 & 0.89 \\
\hline 8 & C22 avg & 27.5427 & 145.4203 & 26.8193 & @C22 avg & 1.34 & 29.5081 & 0.92 \\
\hline 9 & C23 "A" & 71.9809 & 94.1359 & 74.7603 & @C23 "A" & 3.35 & 26.7438 & 3.62 \\
\hline 10 & C23 "A" & as above & as above & as above & @C23 "A" & 2.83 & 27.7606 & 2.63 \\
\hline 11 & C24 "A" & 69.0679 & 98.2404 & 70.9234 & @C24 "A" & 2.21 & 27.7890 & 2.60 \\
\hline 12 & C24 "A" & as above & as above & as above & @C24 "A" & 3.27 & 26.8659 & 3.50 \\
\hline 13 & C26 avg & 42.3909 & 128.9878 & 42.1805 & @C26 avg & 3.22 & 27.1225 & 3.25 \\
\hline 14 & C17 "B" & 47.4914 & 122.0891 & 48.6295 & @C17 "B" & 4.85 & 25.2471 & 5.08 \\
\hline 15 & C18 "B" & 19.2774 & 155.6706 & 17.2372 & @C18 "B" & 1.48 & 28.9426 & 1.48 \\
\hline 16 & C19 "B" & 23.8877 & 149.3135 & 23.1799 & @C19 "B" & 1.64 & 28.7314 & 1.68 \\
\hline 17 & C20 "B" & 42.7085 & 128.1996 & 42.9173 & @C20 "B" & 3.35 & 27.3699 & 3.01 \\
\hline 18 & C21 "B" & 27.2742 & 146.3529 & 25.9475 & @C21 "B" & 1.39 & 29.5123 & 0.92 \\
\hline 19 & C23 "B" & 73.8437 & 93.7184 & 75.1506 & @C23 "B" & 3.64 & 26.9336 & 3.44 \\
\hline 20 & C23 "B" & as above & as above & as above & @C23 "B" & 1.93 & 28.8269 & 1.59 \\
\hline 21 & C24 "B" & 69.8763 & 97.6489 & 71.4763 & @C24 "B" & 4.13 & 25.4912 & 4.85 \\
\hline 22 & C24 "B" & as above & as above & as above & @C24 "B" & 1.74 & 28.3986 & 2.01 \\
\hline
\end{tabular}

The quantification of an level of agreement between theoretical and measured 2D spectra (which are expressed in terms of $[\varepsilon(\mathrm{C}) ; \varepsilon(\mathrm{H})]$ and $[\delta(\mathrm{C}) ; \delta(\mathrm{H})]$ pairs, respectively) leads to the following values of key statistical parameters (22 pairs considered) [references 1,2 ]:

$s_{\mathrm{C}}=1.95 \mathrm{ppm} ; s_{\mathrm{H}}=0.248 \mathrm{ppm} ; s_{\mathrm{CH}}=0.229(\mathrm{ppm})^{2}$.

The experiment-to-theory fit of 22 resolved signals of carbons:

$\sigma(C)=-1.0655^{*} \delta(C)+173.88 \mathrm{ppm} ; S D(\mathrm{C})=2.08 \mathrm{ppm}$ (standard deviation).

The experiment-to-theory fit of 22 resolved signals of protons:

$\sigma(\mathrm{H})=-1.0088^{*} \delta(\mathrm{H})+30.405 \mathrm{ppm} ; S D(\mathrm{H})=0.25 \mathrm{ppm}$. 


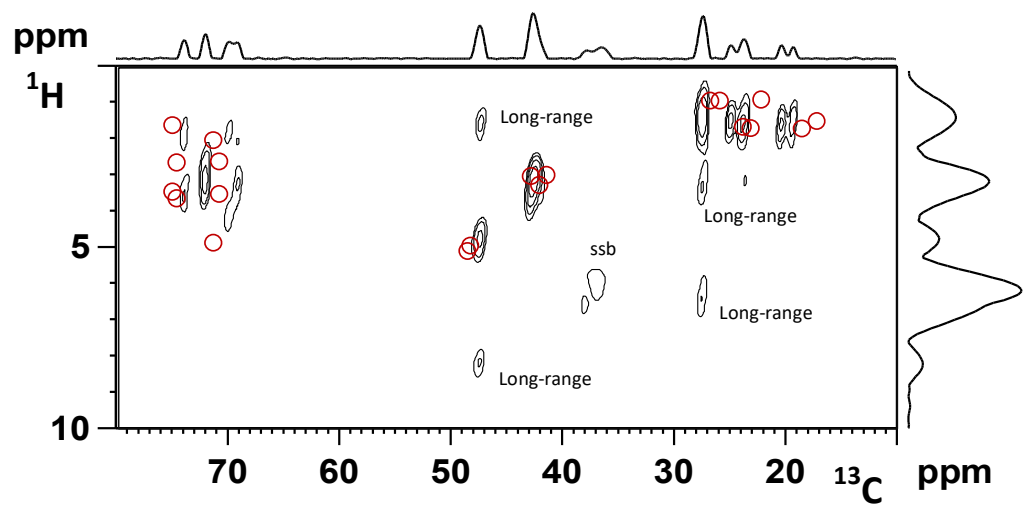

Figure S9. The overlay of experimental ${ }^{1} \mathrm{H}_{-}{ }^{13} \mathrm{C}$ HETCOR NMR spectrum of Selexipag I and calculated theoretical chemical shifts $\varepsilon\left({ }^{1} \mathrm{H}\right)$ and $\varepsilon\left({ }^{13} \mathrm{C}\right)$ for one-bond ${ }^{1} \mathrm{H}_{-}{ }^{13} \mathrm{C}$ pairs (red circles).

\section{References}

1. Czernek, J.; Brus, J. Chem. Phys. Lett. 2014, 586, 56.

2. Czernek, J.; Brus, J. Chem. Phys. Lett. 2014, 608, 334. 